\title{
Laboreal
}

Volume $9 \mathrm{~N}^{\circ} 1$ | 2013

Varia

\section{Modalidades de gestión de la esfera temporal en la conducción de procesos múltiples en situación dinámica : una investigación en empresas hortícolas}

Modalidades de gestão do meio temporal em uma condução de processos múltiplos em situação dinâmica : uma pesquisa em empresas de horticultura Modalités de gestion du milieu temporel dans une conduite de processus multiples en situation dynamique : une recherche dans des entreprises horticoles Management modalities of the temporal dimension in conducting multiple processes in a dynamic situation: a research at horticultural enterprises

\section{Valérie Zara-Meylan}

Traductor: Maria Isabel Muñoz

\section{(2) OpenEdition}

\section{Journals}

Edición electrónica

URL: http://journals.openedition.org/laboreal/6201

DOI: $10.4000 /$ laboreal.6201

ISSN: 1646-5237

\section{Editor}

Universidade do Porto

Referencia electrónica

Valérie Zara-Meylan, « Modalidades de gestión de la esfera temporal en la conducción de procesos múltiples en situación dinámica : una investigación en empresas hortícolas », Laboreal [En línea], Volume 9 N$^{0} 1$ | 2013, Publicado el 01 julio 2013, consultado el 24 septiembre 2020. URL : http:// journals.openedition.org/laboreal/6201 ; DOI : https://doi.org/10.4000/laboreal.6201

Este documento fue generado automáticamente el 24 septiembre 2020.

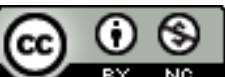

Laboreal está licenciado com uma Licença Creative Commons - Atribuição-NãoComercial 4.0 Internacional. 


\section{Modalidades de gestión de la esfera temporal en la conducción de procesos múltiples en situación dinámica : una investigación en empresas hortícolas}

Modalidades de gestão do meio temporal em uma condução de processos múltiplos em situação dinâmica : uma pesquisa em empresas de horticultura Modalités de gestion du milieu temporel dans une conduite de processus multiples en situation dynamique : une recherche dans des entreprises horticoles Management modalities of the temporal dimension in conducting multiple processes in a dynamic situation: a research at horticultural enterprises

\section{Valérie Zara-Meylan}

Tradución : Maria Isabel Muñoz

\section{REFERENCIA}

Zara-Meylan, V. (2012). Modalités de gestion du milieu temporel dans une conduite de processus multiples en situation dynamique : une recherche dans des entreprises horticoles. Thèse de doctorat en Ergonomie, préparée au Créapt, S. Volkoff et V. Pueyo (dir.). Noisy-le-Grand: Cnam, CEE.

\section{NOTA DEL EDITOR}

Manuscrito recibido en : febrero/2013

Aceptado tras peritaje : mayo/2013 
1 La presente tesis trata de las modalidades de gestión temporal de la actividad en situaciones dinámicas que conllevan procesos múltiples, en nuestro caso se trata de plantas ornamentales en el sector hortícola (Zara-Meylan, 2012). El objetivo de esta producción es el de procurar a las plantas un punto de maduración con una calidad esperada, escalonando los diferentes lotes según la programación de ventas de la empresa. Sin embargo, en sistemas abiertos a influencias exteriores, las condiciones de cultivo son controlables sólo parcialmente y el objetivo fijado es difícil de conseguir. A esto se añade que la actual lógica de racionalización parece estar en contradicción con las necesidades de una producción perecedera que debe ser mantenida en condiciones siempre variables.

2 Desde una perspectiva ergonómica, el cuestionamiento de esta investigación es el de identificar cómo los trabajadores y en particular los jefes de cultivo responsables de los equipos de producción, gestionan las múltiples exigencias temporales presentes en la actividad. Se trata de comprender cómo integran los diferentes riesgos que estas exigencias temporales presentan para una producción eficaz, para ellos mismos y para los obreros que dirigen. Además, se analizan las discordancias entre las exigencias relativas a diferentes lógicas y la lógica del trabajo.

\section{Horticultura y viveros, las dificultades de las pequeñas empresas en búsqueda de racionalización}

3 La presente investigación ha sido realizada en empresas hortícolas y en viveros a lo largo tres proyectos de colaboración ${ }^{[1]}$, que pretenden dar respuesta a problemas de producción y de riesgos laborales comunicados por organismos profesionales y paritarios. En este sector cada vez más competitivo, los dirigentes de las empresas que participan en las investigaciones insisten además en la frágil situación económica de sus estructuras a pesar de una modernización y una mecanización recientes (irrigación, central climática, persianas en los invernaderos, máquinas de replantado, ayudas a la manipulación de cargas, etc.). A raíz de reflexiones en el sector, se han promovido políticas comerciales más ofensivas y se han difundido lógicas de burocratización y de rígido control temporal. Todo ello se salda con un incremento de exigencias de reactividad y de polivalencia, y con modos más rígidos de prescripción y de evaluación del trabajo, en particular con la introducción de medidas de cadencia (Pueyo \& ZaraMeylan, 2012). Sin embargo, estas transformaciones no han permitido la estabilización de la producción y persisten las “ estimaciones de la calidad " con pérdidas de clientes. Las restricciones médicas de aptitud laboral complican, según los dirigentes, la distribución de tareas, y llevan a interrogantes sobre las competencias de los empleados, en particular en relación a puestos clave, tales como el de jefe de cultivo.

4 Estas situaciones pueden asemejarse a las de otros sectores, donde una intensificación del trabajo se traduce en marañas temporales de cada vez más complejas (Askenazy, Cartron, de Coninck, \& Gollac, 2006 ; Volkoff, 2008, 2012). En horticultura, se observa efectivamente una acumulación de exigencias, en particular temporales, cuya asociación es para los trabajadores, difícil de mantener. Estas tensiones atañen particularmente a los jefes de cultivo a cargo de la organización y del seguimiento del trabajo, en organizaciones que esperan mucho de ellos. 


\section{La dirección del cultivo : una articulación específica de exigencias temporales}

5 Como responsables de la producción de una explotación y como managers de proximidad, la misión de los jefes de cultivo es la de alcanzar los objetivos fijados por el dirigente en términos de cantidad, calidad y de plazos, siempre controlando los riesgos para la producción y para los trabajadores. Para dirigir el desarrollo de productos vivos y frágiles, su trabajo requiere de conocimientos altamente especializados. Los jefes de cultivo se encuentran en relación con el dirigente, del que reciben las consignas y a quien rinden cuentas, y en relación además con los trabajadores cuyo trabajo organizan, un trabajo técnico en el que ellos mismos están comprometidos. Además, diversos actores (proveedores, clientes) pueden influir en las actividades de los jefes de cultivo.

6 La tesis defendida es que los jefes de cultivo, en la complejidad y en la dinámica de sus situaciones, identifican y gestionan diferentes exigencias temporales entremezcladas. Retomando los conceptos de W. Grossin, analizamos estas exigencias temporales en términos de proceso a seguir y de "marcos temporales " que ellos gestionan en su “ esfera temporal » de trabajo. Además de precisar estos conceptos, demostramos cómo esta gestión integra las tensiones y los riesgos de la actividad, y la forma en que esta gestión se puede complicar et incluso obstaculizar a causa de ciertas configuraciones de marcos temporales.

\section{Un movimiento metodológico de despliegue de varios aspectos temporales}

7 El desarrollo de esta tesis se basa en un despliegue de las condiciones temporales del trabajo de los jefes de cultivo, en tres fases.

- Una fase exploratoria basada en el análisis de la organización de la producción y de las condiciones temporales del trabajo de los jefes de cultivo, en nueve explotaciones de seis empresas. Se precisan los conceptos de proceso, marcos y esfera temporales.

- Después, los procesos y los marcos temporales son examinados en diferentes ensambladuras. A partir de entrevistas semi-directivas con cinco jefes de cultivo, identificamos las acomodaciones y las configuraciones portadoras de puntos de referencia en su esfera temporal. Algunas configuraciones son particularmente temidas a causa de riesgos difíciles de manejar.

- A continuación, a partir de observaciones sistemáticas de varios días de trabajo, se examinan las actividades conexas de dos jefes de cultivo. Analizamos la gestión dinámica de tramas de marcos temporales en sus esferas temporales. 


\section{Los procesos y los marcos temporales gestionados en la esfera temporal}

9 La horticultura se aparenta a una gestión de procesos tal y como se describe en investigaciones de situaciones dinámicas (Amalberti, 2001 ; Cellier, De Keyser, \& Valot, 1996 ; Pueyo, 2000). Este trabajo se caracteriza por la complejidad, la variabilidad y la incertitud de unos sistemas de producción siempre falibles (Norros, 2004 ; Owen, 2009). Se presentan además el cúmulo y la articulación específicos de condiciones temporales examinados en términos de procesos, en un sentido amplio, así como de marcos temporales.

Los jefes de cultivo tienen que gestionar diferentes procesos que no son únicamente relativos a las plantas, y que constituyen cada uno "un conjunto de fenómenos organizados en el tiempo" (Mendez et al., 2010, p.7). Se distinguen tres tipos de procesos:

- Los procesos de producción a respetar, tal y como son clásicamente definidos en una situación dinámica, se refieren aquí a decenas, incluso centenas de lotes paulatinos, que deben ser respetados a la vez para extender la oferta comercial en las diferentes estaciones. Los procesos son largos, se extienden a varios meses si se trata de plantas con flores y a varios años si se trata de arbustos y de árboles. Cada lote (plantas de la misma variedad en el mismo estadío de producción) presenta necesidades específicas y necesita de cuidados a largo plazo (riegos, podas, intrants, etc.), a pesar de todo ello, el estado de las plantas es inestable. Ya estén los cultivos fuera del suelo o bien en la tierra (grandes árboles), su evolución presenta estados límites e irreversibles que requieren sucesivos diagnósticos o, a veces, acciones urgentes.

- Las condiciones biosféricas (Shippers, 1997) son un conjunto de condiciones climáticas o bioquimicas de cultivo (temperatura, higrometría, iluminacion, confinamiento del aire, etc.), controlables según la especificidad de cada parcela, (orientación, modo de riego, etc.). Ya sea en el exterior o bajo abrigo, la biosfera es solo parcialmente controlable, pues siempre está sujeta variaciones externas (clima, plagas de insectos, etc.). Además, los imprevistos técnicos que la afectan son numerosos (cortes de riego, persianas de invernaderos bloqueadas...). Así, previsiones meteorológicas, diagnósticos globales o locales, anticipación e intervenciones rápidas se hacen necesarios.

- El proceso de trabajo que ha de ser realizado es otro fenómeno organizado, es objeto del trabajo del jefe de cultivo. Este proceso, cuya representación es elaborada a partir de la experiencia (Teiger, 1993; Weill-Fassina, 1990), requiere un trabajo de organización (de Terssac, 2002) compuesto de la articulación de acciones técnicas y sociales entre actores de lógicas distintas, a menudo difícilmente compatibles (obreros, dirigente, y también comercial, logística, etc. ).

11 Otras condiciones temporales del trabajo de los jefes de cultivo se abordan en términos de marcos temporales más o menos rígidos, tal y como han sido inicialmente conceptualizados en trabajos de ecología temporal (Grossin, 1996). Se distinguen en horticultura :

- Los marcos de planificación de cultivo fijan el calendario de puestas en cultivo y el calendario de ventas. En cada empresa, guían fuertemente la actividad, incluso si a veces son solo formalizados parcialmente, y son continuamente modificados por el desistimiento de los 
clientes, o por la decisión del dirigente de abrirse, a veces con poca anticipación, a nuevos mercados.

- -Los marcos de la organización del conjunto de la producción están vinculados a los horarios y al calendario de semanas de trabajo, también a la cadencia de las máquinas, al sistema de gestión y a las normas que imponen articulaciones y medidas, a los efectivos presentes y a los espacios que influyen en la distribución de los trabajos, en su duración y en los desplazamientos.

- Los marcos temporales denominados encerrados, son relativos a condiciones de trabajo más locales, que pueden ser diferentes según las tareas y el puesto. Se derivan de instrucciones y de procedimientos, así como de articulaciones internas a la empresa (con el comercial, la logística) y externas (con los proveedores o los clientes).

12 Según la situación, estos procesos y estos marcos se traducen en diferentes tipos de requisitos (desencadenantes, topes, duración, paréntesis, ritmos y cadencias impuestas). Delimitan, constriñen, así como estructuran y apoyan la actividad. Desde una perspectiva ergonómica, son a la vez exigencias y recursos en la actividad. Los jefes de cultivo los vinculan para constituir su esfera temporal.

Para precisar la noción de esfera temporal de trabajo que desarrollamos, examinemos los ensamblajes de marcos temporales entremezclados que lo componen, los cuales tienen formas de acciones específicas en la actividad. En primer lugar describimos las acomodaciones y las configuraciones temporales, y después las tramas de marcos temporales.

\section{Ensamblar los marcos temporales en acomodaciones y configuraciones}

Las acomodaciones estacionales asocian los marcos temporales de gestión de cultivos (puestas en cultivo y venta en determinados periodos del calendario) con los principales procesos de crecimiento de las plantas. Cinco tipos de acomodaciones, no exhaustivos, emergen de nuestro análisis: las acomodaciones mono-estacionales (un marco de cultivo y uno de venta), bi-estacionales (dos marcos de cultivo se superponen con los de venta), plurianuales (árboles atendidos durante años con ventas más bien invernales), mixtos (gestión mono o bi-estacional, con árboles), y en cascada (las puestas en cultivo y las ventas se suceden y se superponen; para las plantas con flores, por ejemplo).

Las configuraciones están compuestas por relaciones dinámicas (Elias, 1993 ; Grossin, 1996), periódicas o puntuales, entre marcos temporales y entre la organización del proceso de trabajo. Algunas configuraciones esquematizan situaciones habituales, corrientes y a veces incluso repetitivas, que se tienen que gestionar; otras veces se refieren a situaciones destacadas en por su coyuntura o por su evolución.

Algunas configuraciones son particularmente temidas por los jefes de cultivo, a causa de dinámicas turbulentas portadoras de exigencias contradictorias y de riesgos que no pueden evitar (Pueyo, 2012; Valot, 1998). Uno de ellos describe por ejemplo una configuración temida de primavera, que asocia las exigencias de la biosfera, del trabajo y de los procesos relativos a las plantas, en este momento los marcos de venta pasan a ser imperativos. A causa de una acomodación bi-estacional de la dirección de su empresa, el jefe de cultivo deberá poner en cultivo nuevas plantas, al mismo tiempo 
que un deshierbe suplementario se impone. Esta configuración se salda con riesgos crecientes para la calidad de la producción cuyos cuidados son retardados, con dolores (dedos, rodillas, espalda), con lesiones (rozaduras, picaduras) y con fatiga acumulada por todo el equipo.

Los análisis muestran distintos modos de acción en los marcos temporales. En el ejemplo relatado, el jefe de cultura refuerza los marcos de los cuidados (poda, emplazamiento de rodrigones, deshierbado...) "un día cada uno", aunque el trabajo desborde. Para desplazar un trabajo previsto en ese momento, prueba con comentarios como "Los bambúes, ¿no vamos a hacer también de 6 litros? Porque el año pasado... ». Además, de este modo alerta sobre las consecuencias de una multiplicación de pequeños lotes, lo que complica la articulación de los diferentes cuidados a las plantas y puede aumentar el retraso. Sin embargo, a veces, si sobreviene una avería o si los pedidos tardan, no tiene más remedio que adaptarse: "voy corriendo, corriendo... ", explica.

De este modo, los riesgos no están únicamente vinculados a evoluciones indeseadas en procesos. También dependen de la ausencia de posibilidades de integrar las variaciones y los imprevistos en la gestión de las situaciones (Zara-Meylan, 2013).

\section{Tejer y articular las tramas de los marcos temporales}

En su actividad, los jefes de cultivo no prevén solamente los acontecimientos y las evoluciones, ni reaccionan únicamente a lo que ocurre (biosfera, solicitudes de clientes...) a partir de determinados criterios (una emergencia, un contratiempo...). Manejan un conjunto de procesos y de marcos temporales de difícil conciliación, pero ellos los organizan y tejen tramas en espacios más o menos amplios. El análisis de la actividad muestra una sucesión de episodios durante los cuales explotan una verdadera gestión activa y prospectiva de su esfera temporal, "pensando " la articulación de tramas complejas aunque inteligibles (Le Moigne, 2009 ; Morin \& Le Moigne, 1999).

La gestión de la esfera temporal por los jefes de cultivo debe ser comprendida más allá de la acción del momento, en un conjunto de otras acciones efectuadas (Mead, 1967, 1st edt 1934), y en su inscripción en un seguimiento técnico y de interacciones sociales (Joas, 2004, 1ère édt 1999; Pueyo, 2010). Este conjunto de acciones no está orientado únicamente por objetivos directamente operativos, objetivos cuya realización es siempre incierta en horticultura. También responde a la renovación y al aumento de opciones en sus acciones.

\section{Los marcos y sus ensamblajes en la actividad, para transformar el enfoque de la organización sobre los riesgos}

Considerando la acción temporal como una dimensión fundamental de la actividad, esta investigación propone una estructura de análisis de exigencias del trabajo que favorezca a los trabajadores la integración de riesgos en la gestión de situaciones.

Estas aportaciones se discuten bajo una perspectiva de investigación y de acción, ésta última necesita en particular de colaboraciones con los expertos en prevención de 
riesgos laborales y en formación, en este sector y en pequeñas empresas. En el transcurso de nuestras investigaciones, este encuadre ha favorecido la visibilidad del trabajo y de su sentido para la organización y para los empleados, a partir del debate sobre las fronteras del espacio aceptable. Esto ha permitido acompañar la transformación del enfoque de la organización sobre los riesgos.

Las perspectivas son la mejora del estatus de las acomodaciones, de las configuraciones y de las tramas de marcos temporales, como soportes útiles para la acción sobre el trabajo. Futuros trabajos podrían versar sobre el desarrollo, con la experiencia, de competencias de gestión temporal en la dinámica de la actividad en otras situaciones profesionales, y en perspectivas socio-históricas más amplias.

\section{BIBLIOGRAFÍA}

Amalberti, R. (2001). La maîtrise des situations dynamiques. Psychologie Française, 46(2), 105-117.

Askenazy, P., Cartron, D., de Coninck, F., \& Gollac, M. (Eds.). (2006). Organisation et intensité du travail. Toulouse Octarès.

Cellier, J.-M., De Keyser, V., \& Valot, C. (1996). La gestion du temps dans les environnements dynamiques. Paris : PUF.

Elias, N. (1993). Engagement et distanciation. Paris : Fayard.

Grossin, W. (1996). Pour une science des temps. Introduction à l'écologie temporelle. Toulouse : Octarès. Joas, H. (2004, 1ère édt 1999). La créativité de l'agir (P. Rusch, Trans.). Paris : Edition du Cerf. Le Moigne, J.-L. (2009). L'Intelligence de l'Action appelle l'exercice de la Pensée Complexe. Pragmatique et Epistémique sont inséparables. Conférence introductive. Synergies Monde, 6, 23-48.

Mead, G. H. (1967, 1st edt 1934). Mind, Self and Society from the Standpoint of a Social Behaviorist (Vol. 1). Chicago: University of Chicago Press.

Mendez, A., Bidart, C., Brochier, D., Correia, M., Garnier, J., Gilson, A., et al. (2010). Chapitre introductif. Vers un système d'analyse des processus. In A. Mendez (Ed.), Processus. Concepts et méthode pour l'analyse temporelle en sciences sociales (pp. 11-26). Louvain-la-Neuve, Belgique : Bruyant-Academia.

Morin, E., \& Le Moigne, J.-L. (1999). L'intelligence de la complexité. Paris : L'Harmattan.

Norros, L. (2004). Acting under uncertainty. The core task analysis in ecological study of work: Espoo 2004. VTT Publications 546.

Owen, C. (2009). Accomplishing Reliability within Fallible Systems. In C. Owen, P. Béguin \& G. Wackers (Eds.), Risky Work Environments. Reappraising Human Work Within Fallible Systems (pp. 99-103). Farnham (G.B.): Ashgate.

Pueyo, V. (2000). La traque des dérives : expérience et maîtrise du temps, les atouts des “ anciens » dans une tâche d'autocontrôle. Travail et Emploi, 84,63-73. 
Pueyo, V. (2010). Quelle gestion de la sécurité dans une structure temporelle incertaine ? La gestion des risques de pépiniéristes Actes du séminaire CREAPT Ages et Travail 2008. Rapport de recherche CEE $n^{\circ}$ 58. Expérience professionnelle et gestion de la sécurité au travail (Vol. www.ceerecherche.fr/, pp. 105-117). Paris : Centre d'Etudes de l'Emploi.

Pueyo, V. (2012). Quand la gestion des risques est en péril chez les fondeurs. In C. Gaudart, A.-F. Molinié \& V. Pueyo (Eds.), La vie professionnelle : âge, expérience et santé à l'épreuve des conditions de travail (pp. 257-284). Octarès.

Pueyo, V., \& Zara-Meylan, V. (2012). Impacts d'outils de gestion sur la conduite de cultures en pépinière. @ctivités, 9(1), 1-20 http://www.activites.org.

Shippers, T. K. (1997). Perceptions et domestications du cycle annuel, une approche ethnoécologique Les temps de l'environnement (Vol. Session 3, 4 \& 5, Conférences plénières, pp. 195-200). Toulouse.

Teiger, C. (1993). L'approche ergonomique : du travail humain à l'activité des hommes et des femmes au travail. Education Permanente, 116, 71-96.

de Terssac, G. (2002). Le travail d'organisation comme facteur de performance. In G. De Terssac (Ed.), Le Travail : une aventure collective. Recueil de textes (pp. 149-159). Toulouse : Octarès.

Valot, C. (1998). Métacognition et connaissances métacognitives. Intérêt pour l'ergonomie. Unpublished Thèse de doctorat de nouveau régime. Spécialité Ergonomie, Toulouse.

Volkoff, S. (2008). L'intensification disperse les problèmes de santé. In G. De Terssac, C. SaintMartin \& C. Thébaud (Eds.), Travail, organisation, santé: une question de précarité. Toulouse : Octarès.

Volkoff, S. (2012). Le travail, en évolutions. In C. Gaudart, A.-F. Molinié \& V. Pueyo (Eds.), La vie professionnelle : âge, expérience et santé à l'épreuve des conditions de travail (pp. 31-42). Toulouse.

Weill-Fassina, A. (1990). Activités et compétences professionnelles. In M. Dadoy, C. Henri, B. Hillau, G. de Terssac, J.-F. Troussier \& A. Weill-Fassina (Eds.), Les analyses du travail ; enjeux et formes (Vol. 54, pp. 145-148). Paris : Cahiers du Cereq.

Zara-Meylan, V. (2013). Faire face aux imprévus sans être pris au dépourvu : le cas des chefs de culture dans de petites entreprises horticoles. Sociologies Pratiques, 26. Retirado de http:// www.cairn.info/revue-sociologies-pratiques.htm.

\section{NOTAS}

1. Los análisis se basan en investigaciones llevadas a cabo por un equipo de ergónomos del CREAPT en colaboración con organismos de formación y de prevención que intervienen de forma trasversal en el sector agrícola y con la participación de empresas hortícolas y viveros, a lo largo de tres proyectos :

- « Agriquadra ", un proyecto Equal sobre los vínculos del envejecimiento y el trabajo en los sectores agrícolas, llevado a cabo en colaboración con el Fondo nacional de Seguros Formación de los Salariados de Explotaciones y empresas Agrícolas (Fonds national d'Assurance Formation des Salariés des Exploitations et entreprises Agricoles -FAFSEA-), la Caja Central de la Mutualidad Social Agrícola (Caisse Centrale de la Mutualité Sociale Agricole-CCMSA-) y la Federación Nacional de Productores de Horticultura y Viveros (Fédération Nationale des Producteurs de l'Horticulture et des Pépinières -FNPHP-), de 2006 à 2008. 
- « La evaluación de riesgos laborales en los viveros », en respuesta a una solicitud del Servicio de Reglamento y de Trabajo del Ministerio de Agricultura y Pesca (Bureau Réglementation et Travail du Ministère de l'Agriculture et de la Pêche) en 2007.

- "Prevención de riesgos y preocupaciones sobre la producción de pequeñas empresas », en colaboración con la Federación des MSA (mutualidades sociales agrícolas) del Languedoc y con la participación de empresas hortícolas y de viveros de esta región, en 2009 y 2010.

\section{AUTORES}

\section{VALÉRIE ZARA-MEYLAN}

Centre d'Etudes de l'Emploi, Centre de Recherches sur l'Expérience, l'Age et les Populations au Travail, , Immeuble Le Descartes 1, 29 promenade Michel Simon, 93166 Noisy-le-Grand, France valerie.meylan@cee-recherche.fr 